\title{
Research and Development of Practical Teaching Quality Standards in Application-Oriented Undergraduate
}

\author{
CHEN Zhi-wu, ${ }^{1, a,{ }^{*}, \text { LUO Zhi }}{ }^{2, b}$ \\ ${ }^{1}$ Finance \& Economics College , Hunan University of Technology, Zhuzhou,Hunan,CHINA \\ ${ }^{2}$ Finance \& Economics College , Hunan University of Technology, Zhuzhou,Hunan,CHINA \\ achenzhiwu08055@163.com, bj cjluozhi@163.com \\ ${ }^{*}$ Corresponding author CHEN Zhi-wu
}

\begin{abstract}
Practical teaching is an important link in cultivating of application-oriented undergraduate talents. The school quality standard is of great significance for the specification and evaluation of practice teaching and pointing out the direction for running school. Through the research on the connotation of standard and the influencing factors on quality standard of practical teaching,the establishment of application-oriented undergraduate practical teaching quality standard should be standardized from input, process and output of practical teaching. At the same time,the standard establishment should follow operability and classification principle as well as the normative principles of the standard text.
\end{abstract}

Keywords: Application-oriented undergraduate; Practical Teaching;Quality Standards

\section{应用型本科实践教学质量标准研制}

\author{
陈支武 ${ }^{1, a, *}$ ，罗志 ${ }^{2, b}$ \\ ${ }^{1}$ 湖南工业大学财经学院, 株洲, 湖南, 中国 \\ ${ }^{2}$ 湖南工业大学财经学院, 株洲, 湖南, 中国 \\ achenzhiwu08055@163.com, b cjluozhi@163.com \\ 通讯作者陈支武
}

摘要: 实践教学是应用型本科人才培养 的重要环节。校内质量标准对于规范与评价 实践教学以及指明学校的办学方向具有重要 意义。通过对标准内涵的规范研究和实践教
学质量标准的影响因素研究, 认为应用型本 科实践教学质量校内标准制订应从实践教学 投入、过程与教学输出三个维度进行规范。 同时, 质量标准在制订时应遵循可操作性与 
分类的原则以及标准文本的规范性。

关键词: 应用型本科; 实践教学; 质量标 准

\section{1 引言}

提高质量，是当前高等教育改革最核心 最紧迫的任务。《国家中长期教育改革和发 展规划纲要（2010--2020 年）》（以下简称 “教育规划纲要” ) 明确提出提高高等教育 质量和人才培养质量 ${ }^{[1]}$ 。2011 年 7 月教育部 财政部发布的 “教育部 财政部关于 ‘十二五 期间实施高等学校本科教学质量与教学改革 工程” 的意见” (教高 [2011]6 号)。意见中 关于 “建设内容” 的第一条就是 “质量标准 建设”，其内容是 “组织研究制定覆盖所有 专业类的教学质量国家标准, 推动省级教育 行政部门、行业组织和高校联合制定相应的 专业教学质量标准, 形成我国高等教育教学 质量标准体系” ${ }^{[2]}$ 。2012 年 3 月教育部又出 台文件 “教育部关于全面提高高等教育质量 若干意见” (教高 [2012]4 号), 意见提出 “强 化实践育人环节。制定加强高校实践育人工 作的办法, 结合专业特点和人才培养要求, 分类制订实践教学标准” ${ }^{[3]}$ 。本文基于以上 背景, 对应用型本科实践教学质量校内标准 问题进行研究与探讨。

\section{2 实践教学质量标准的基本内涵及研制意义}

\section{1 实践教学质量标准的基本内涵}

所谓标准, 通常可以这样表述: “为了 在一定的范围内获得最佳秩序, 经协商一致 制定并由公认机构批准，共同使用和重复使 用的一种规范性文件。” ${ }^{[4]}$ 。目前, 人们往往 将 “标准” 与 “质量” 两者联系起来, 认为 它是为保证活动的质量和效果而对活动目 标、活动内容、活动程序等作出的一种规范, 在形式上表现为一种文本性的规定。标准具 有共识性、规范性、分类性、主体性与客体 性等特点。所谓共识性, 是指在某一行业推 行的标准是经大家协商一致的, 共同认可的, 以此作为产品质量控制标准, 以保证产品的 一致互换与简化; 所谓规范性是指标准的内 容与形态以及标准制定的程序是规范的; 而 分类性则是指; 某一标准都是在特定的类型、
类别范围内推行的，不同的类型、类别就应 有不同的标准。标准的主体性是指, 标准的 设立和运用取决于标准制定者和运用者的价 值观念和价值标准，制定者不同，标准也就 不同, 同一标准运用者不同其理解就有区别。 而标准的客体性则主要是指标准总是从客观 存在中抽象出来, 或以一客观状态作为原型 的 ${ }^{[5]}$ 。

依照对标准的这些理解, 并套用标准定 义, 实践教学质量标准则可以定义为: 它是 由公认机构制定和批准的, 关于实践教学活 动或活动结果并反映实践教学质量的指标和 参数的规范性文件。实践教学质量标准是标 准的下位概念, 具有标准的一般特性, 为此, 我们能从标准的一般特性出发来进一步认识 实践教学质量标准。首先从标准的共识性来 看, 没有共识就没有标准。实践教学相对于 理论教学来说, 由于其教学环境的非真实性、 教学项目的虚似性和教学活动过程的弱控制 性等特点, 使得人们对什么是实践教学质量, 实践教学质量衡量标准是什么, 众说纷纭。 难以达成共识, 但在一校范围之内这种共识 却比较容易达成。

其次, 从标准的规范性上看, 标准的内 容和形态都具有很强的专业性, 因此, 为保 证实践教学质量标准的规范性, 在研制过程 中需要广泛吸收各种专业人员参加, 听取不 同领域专家的意见，包括教学一线的教师、 教学管理人员、质量管理专家及标准化科研 人员等。学校要组织相关专业人员进行研究 和论证, 从程序上和内容上保证质量标准的 规范性。

第三，从标准的分类性看，标准总是在 一定范围内的标准，不同类型不同层次的本 科院校应有不同的实践教学质量标准。目前, 我国高校的人才培养类型与层次尽管尚有争 议, 但普通认为存在学术型和应用型两种基 本类型, 以及研究型、教学研究型以及教学 型三个层次。学术型人才主要从事科学研究 与发现客观规律的工作，而应用型人才的是 擅长技术的应用, 能解决生产实际中的具体 问题，是现代技术的应用者与实现者 ${ }^{[6]}$ 。不 同高校由于其人才培养规格的不同, 实践教 学的质量标准应当分别研制。此外, 实践教 学质量标准除按人才培养类型与层次分别研 
制以外, 区分不同的实践教学类型制订标准 也应是分类制订的应有之意。

第四, 从标准的主体性与客体性来看, 质量标准在教育环境里, 是指一种对目标成 果的水平和这个成果价值的一种判断, 且这 种判断也是根据某种价值标准的, 或是隐含 着对目标、活动或者成果的预期 ${ }^{[5]}$ 。也就是 说, 不同的标准制订者对实践教学的期望不 同, 价值取向各异, 其标准就会有区别。实 践教学质量标准的客体性是指标准应反映了 实践教学的内在结构和实际状态。实践教学 与理论教学一样, 包括教学输入、过程与教 学输出。相应的, 实践教学的质量标准也应 包括教学输入标准、教学过程与教学输出标 准。这些内容一方面客观反映了实践教学的 内在结构, 另一方面了也反映了人们对它们 关系的认识和把握。

\section{2 研制意义}

高校层面的实践教学质量标准的研制至 少具有以下几个方面的重要意义。

（1）引导与规范作用。标准本身就是价 值判断的结果, 无论对教师还是对学生都具 有很好的价值引导作用。标准能够将教师的 教学行为和学生的学习行为引导到标准所确 定的价值轨道上来。与此同时, 质量标准对 于规范教师的实践教学行为具有重要作用, 长期以来, 高校的实践教学行为, “千校千 面”, 就是同一高校, 也是 “千师千面”, 高 校内部严格按质量标准组织教学则能在相当 程度上规范这种混乱局面。

(2) 为实践教学的评价提供依据。当 前, 很多高校建立了内部教学质量评价制度, 其评价方法或体系大多借用教育部原本科教 学水平评估指标体系, 如对实践教学的评价 要求是 “时间有保证、措施完善、效果好; 注意内容更新, 体系设计科学合理, 符合培 养目标要求” 等。这种评价宏观, 而且不同 的人不同的理解。导致评价结果缺乏科学性, 而实践教学质量标准的制订则为高校的实践 教学评价提供了强有力的科学依据。

（3）质量标准对于明确学校办学方向, 增强学校办学特色具有重要意义。质量标准 按其层次不同可分为国家标准、地区标准、 学校标准与个体标准 ${ }^{[7]}$ 。这是高教系统各参
与者不同的利益诉求所导致的, 也是教育质 量多元化的根源。在国家标准与地区标准的 基础上，各高校根据自己学校类型、层次、 专业类型与特色、特长等, 增添学校办学与 专业特色指标不仅能够使学校明确自身的办 学方向和目标, 而且也能增强学校的办学特 色。

\section{3 实践教学质量标准制订的影响因素分析}

\section{1 质量观的差异影响实践教学质量标准的 制订}

质量观不同, 所确定的质量标准及对质 量的检测办法就不同。现有理论研究中有关 质量的研究基本上都是教育质量, 但教育质 量的核心是教学质量, 因此本文将教育质量 的相关理论运用到教学质量的讨论中来。有 关教育质量的观点学者们的提法尽管各不相 同，但概括起来不外乎以下核心观点。一是 “符合标准的质量观”。该观点认为,质量就 是符合预先设定的规格或目标, 衡量教学质 量的标准是教育目的和各级各类学校的培养 目标 ${ }^{[6]}$ 。这一观点认为不同类型、不同层次 高校的人才培养目标定位是不同的,如学术 型高校与应用型高校人才培养目标就不相 同，因此衡量教学质量的标准也应该有差异。 持符合标准的质量观的就会理所当然地重视 客观质量标准的确立和符合规定标准的检 测。

二是 “满足需要的质量观”。ISO9000 族 标准将质量定义为: “一组固有特性满足要求 的程度”, 这里的要求是指顾客和其他相关方 明示的、通常隐含的或必须履行的需求或期 望。因此教育质量可理解为: 教育的质量特 性满足教育消费者要求的程度 ${ }^{[8]}$ 。那么高等 教育的消费者, 或者说顾客是谁呢? 显而易 见, 高等教育的顾客是多元的,不仅包括学 生、家长、用人单位、政府，甚至还包括整 个社会。且这些顾客的需要是不一样的,越能 满足这几类顾客需求质量就越高。持满足需 要的质量观的, 就会重点关注教育消费者, 特别是学生、用人单位等顾客的满意度。

三是 “关注全程的质量观”, 该观点认为, 只关心结果,而无视教育条件和教育过程, 是 难以达成高质量的教育目标的。全面质量管 理理论认为,教育质量是在一个完整有序的 
过程中形成的。这一过程包括了输入、过程 和输出三个最基本的阶段, 并不只是涉及到 教育的产出, 更不是人才培养质量这一种产 出所能替代的。输出质量是由输入质量和过 程质量所决定的, 从质量建设或质量保障的 角度来讲, 改进质量其实质是改进输入质量 和过程质量。持全程质量观, 就会不仅关注 结果, 而且还会关注人才培养方案设计、教育 教学过程、生源质量、办学条件等条件和过 程的质量。

那么, 在应用型本科实践教学质量标准 建立过程中我们应该坚持什么样的质量观 呢? 我们认为科学的质量标准的建立, 应是 几种教育质量观综合作用的结果。首先, 应 该认识到, 不同层次不同类型的高校因为办 校定位, 办校目标的差异, 因而质量标准是 不相同的, 但同一层次同类高校质量则是可 以比较的。也就是说, 从整个国家层面来看, 高等教育质量观是多元的, 但具体到一类高 校某一层次的高校来说, 则应是具体的, 否 则, 会因为质量的多元化的导向不明而导致 随意化 ${ }^{[9]}$ 。其次, 我们应该注意到应用型本 科教育各利益相关方的诉求。在实践教学质 量标准的制订过程中, 相关指标既要有学校 认可的学术标准指标, 又要有学生经过实践 后自身兴趣、特长、品质提高等考核指标, 更要有用人单位等对学生经过实践教学后应 具备的解决实际问题能力的期望指标。再 次, 在实践教学质量标准建立过程中, 我们 应该秉持这种一种观点, 即教学质量是多种 因素综合作用的结果, 其体现不能仅仅以教 学结果一一学生作为唯一的载体, 要坚持改 变过去那种只注意教学结果, 不注意过程的 教学质量评价方法, 要坚持结果与过程的统 一, 要坚持质量是学出来的, 也是教出来的, 还是管理出来的观点, 因此, 实践教学质量 标准的建立既要有教学结果的指标。也要有 教学投入和教学过程的考核指标。

\section{2 应用型人才特点对实践教学的特殊要求 影响质量标准制订}

实践教学质量标准应用型本科不同于学 术型本科, 其影响因素除其秉持的质量观有 区别以外, 还有其培养的人才特点的不同。 2007 年 5 月, 在上海召开的应用型本科教育
学术研讨会上, 代表们一致认为, 应用型本 科的办学理念和发展定位为培养直接面向市 场和生产一线的高级应用性人才的本科高等 院校。应用型本科人才不同于学术型本科人 才和高职高专人才, 是一种新型的人才类型。 “重应用、重实践、重解决实际问题” 是应 用型本科人才的基本特征, 该类人才具有较 强的技术思维能力, 擅长技术的应用, 具备较 强的解决生产和管理中实际问题的能力, 而 这些能力正是应用型本科人才区别于学术型 本科人才的根本标准 ${ }^{[10]}$ 。

应用型人才培养方案的课程体系中, 实 践教学环节正是培养学生实践能力、应用能 力、创新精神的基本途径, 它将学生课堂知 识的学习与实际的生产场景联系在一起; 将 书本的理论知识与生产过程中的项目相结 合; 将学生的考核与分析和解决实际问题的 能力相结合。课程体系通过诸如实验教学、 认识实习、生产实习, 校内实训、毕业实习、 毕业论文等各类实践教学, 体现了应用型本 科教学的鲜明特色 ${ }^{[11]}$ 。

应用型本科人才特点对实践教学的这些 特殊要求, 我们在制订实践教学质量标准的 过程中应当充分考虑到, 在质量形成的全过 程, 应设计相应的技术指标或指标体系来规 范各个阶段的条件与行为, 反映人才的应用 能力、创新能力等。

\section{4 实践教学质量标准需规范的内容及需注意 的问题}

\section{1 实践教学质量标准需规范的内容}

标准从其本质属性来看属于技术规范。 而实践教学提供的是一种服务, 若将两者结 合, 就要考虑这种服务中的技术指标和技术 指标体系。提供实践教学服务需要具备一定 的物质基础, 如各种实践教学设备与工具等。 为提高实践教学的质量, 对这一类作为实践 教学投入的装备进行标准和标准化工作是必 须的, 但仅仅将实践教学质量标准制订停留 在实践教学装备这一类硬件上, 是远远不够 的, 还必须对诸如教师资格、教学过程、教 学效果等 “软件” 进行标准化。才能真正达 到提高质量之目的。相对于实践教学的 “硬 件” 来说, 实践教学的 “软件” 却不易规范。 因为各高校的教学模式与教学特色不尽相 
同, 而标准要求的却是统一和一致, 按照同 一规范能在越大的范围内实行越好。可见这 样的要求与实践教学质量这种软性目标相 比, 本身就带有一定的矛盾和冲突。因此在 制订实践教学质量标准时, 一定要搞清楚哪 些方面是可以而且应该统一规范的, 哪些内 容则是不能统一而要放开搞活的 ${ }^{[4]}$ 。

本文是站在高校的角度来研究实践教学 质量标准的。因此, 各高校应充分考虑本校 的教学模式以及在实践教学方面的的特色与 专长, 结合国家、地区的质量标准来建立自 己的质量标准。那么应用型本科实践教学质 量标准在制订过程中应规范的内容到底有哪 些呢? 借鉴全面质量管理的思想, 我们认为 实践教学质量不仅是学出来的, 也是教出来 的, 还是管出来的, 因而实践教学质量标准 的建立既要有教学结果的指标。也要有教学 投入和教学过程的考核指标。为此, 实践教 学质量标准的建立所需规范的内容可以从教 学投入、教学过程和教学输出三个方面进行 规范。这样的规范在标准的制订过程中, 思 路是清晰的, 同时也便于标准文本的表述。 各环节需规范的包括但不限于以下内容:

关于实践教学投入: 至少应规范以下内 容。（1）实践教学师资队伍;（2）各类实践 教学的设施条件, 如校内的实验室条件以及 相关实验教学用软件等, 校外实习环境与实 习单位条件、实践教学经费等; (3) 实践教 学用教材; (4) 实践教学管理制度等。

关于实践教学过程。实践教学过程包括 （1）教的过程。包括实践教学内容的界定, 同一实践教学活动不能因指导教师的不同而 在教学内容上存在差异。教学方法与教学手 段。教学文件的统一规范, 如实践教学大纲、 实践教学进程计划、实践教学记录与总结等;

(2) 学的过程。包括学生的学习态度、学习 认知、学习资源的利用、学习方法、小组讨 论、实践前的个人学具准备等; (3) 管理的 过程。包括管理人员对实践教学投入、教师 教学过程、各种制度规范和落实等情况进行 的检查等。

关于实践教学输出。即受教育者一一学 生, 在受教育后在知识、能力与素质等方面 应表现出来的成就 ${ }^{[12]}$ 。它是实践教学质量标 准制订过程中的核心内容。相对于教学投入、
教学过程, 教学输出的规范更加不易。对学 生知识、能力与素质不仅要从学术方面, 更 要从用人单位对应用型人才的期望方面, 还 要从学生个人发展方面进行定性或定量规 范。以体现应用型人才的本质特征。

\section{2 实践教学质量标准制订应注意的问题}

实践教学质量标准是一种标准, 那么就 应该遵循标准制定的规则。为此, 应用型本 科实践教学质量标准的制订应根据其特点, 结合各高校特色, 按照《标准化与相关活动 的基本术语及其定义》指南, 以及我国 《标 准化工作导则》的要求制定, 只有这样才能 制定出真正意义上的实践教学质量标准, 进 而才能保证应用型本科实践教学质量的稳步 提高。根据以上分析, 我们认为在实践教学 质量标准制订过程中应注意解决好以下两个 主要问题, 即科学的标准制订原则, 和规范 化的标准文本。

首先, 科学的实践教学质量标准应具有 可操作性。质量标准是教师指导具体实践教 学活动的规范, 是考核学生实践能力的标准 和尺度, 也是高校内部开展教学评价的操作 指南, 因此必须具有可操作性。质量标准所 规范的内容应以条目的形式呈现, 明确实践 教学条件, 所需训练的内容, 具体经过的过 程, 应具备的教学文件以及教学后意欲达到 的目标与效果等。为保证质量标准的可操作 性, 其在制订过程中还必须遵循分类制订原

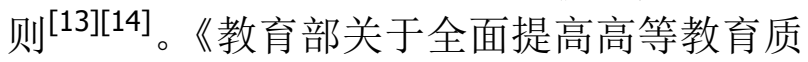
量的若干意见》中也明确提出: “结合专业特 点和人才培养要求, 分类制订实践教学标准” [3]。应用型本科实践教学种类很多, 从教学 组织形式上来看可分为课内和课外实践教 学, 从教学环境上亦可分为校内和校外实践 教学, 以作者所在湖南工业大学经管类实践 教学为例, 可分为实验教学, 认识实习、专 业实习、模拟实习、学年论文、毕业实习、 毕业论文等。只有分类制订质量标准, 才能 使标准发挥其应有的引导、规范和评价作用。

第二, 必须完善实践教学质量标准文本 的规范性。实践教学质量标准文本的规范性 是指制订的质量标准应该符合现代评估理 论、方法与技术要求。标准所使用有语言内 涵要准确, 外延要清晰。无论是对实践教学 
的投入、过程还是输出, 在制订标准时, 尽 可能不使用 “加强、认真、努力、积极、坚 持” 和 “很好、较好、基本能做到” 等表示 程度的弹性语言, 而使用 “是与否”、“对与 错”、“达到与否” 等判断性语言, 以避免参 评者对标准尺度的理解不一, 掌握不准, 而 影响评价的准确性 ${ }^{[4][15]}$ 。

\section{5 结束语}

实践教学是应用型人才培养的关键环 节。实践教学质量的提高高校要做的工作很 多, 而基于高校层面的质量标准制订则是其

\section{致谢}

本文是湖南省 2012 年教改项目: 应用型本科 院校经管类专业实践教学质量标准研究（湘 教通 [2012]401 号) 的阶段性成果之一。

\section{References}

[1] An Outline of the National Middle-Long Term Planning for Educational Reform and

Development(2010-2020) [M].Beijing:People's Publishing House.2010:14.

[2] The Ministry of Education and the Ministry of Finance implementing opinion on 'the Undergraduate Teaching Quality and Teaching Reform Project 'during the 12th 5-year period(Teach high [2011] NO6).

[3] Some sugestions of the Ministry of Education on the overall improvement of the quality of higher education(Teach high[2012]4).

[4] WANG Zhong-min.Reflections on the Education Standards[J].People's

Education.2012,

(12):2-4.

[5] CHEN Yu-xiang.From the standardal connotation to see the establishment of our undergraduate teaching quality standard[J].China Higher Education Research.2007,(7):35-37.

[6] SUN Ze-ping,HE Wan-guo.The Analysis for talent training quality standards of the Newly -built Universities[J].Education exploration.2010,(11):79-80.
中最为重要的工作之一。实践教学质量标准 是关于实践教学活动或活动结果并反映实践 教学质量的指标和参数的规范性文件。应用 型本科实践教学质量标准制订需要考虑其为 生产一线培养 “重实践、重应用” 的高级专 门人才的办学定位。质量标准应按实践教学 类别分类制订, 方具可操作性, 同时应按国 家标准化相关规定规范质量标准文本。可行 的质量标准才能为实践教学质量提高奠定坚 实基础。

[7] ZHAO Ling-li. With talent quality as the core to govern the education quality--concurrently discuss"Six-dimensional four-grade"talent quality standard and monitoring.People's

Education[J].2012,(12):5-9.

[8] CHENG Feng- chun,WEI

Zhe.Re-discussion on Education Quality and Its Standards-From

the Perspective of ISO9000

Series[J].Educational

Research.2012,(6):56-60.

[9] ZHOU Quan-xing.Higher Education

Quality Standard: Characteristics, Orientation of Value and Structural System[J].Jiangsu Higher

Education.2004,(3):8-10.

[10] ZI Kun,PENG Peng-feng, LONG Zhi-jun, YANG Zhi-yong.Research on the basic features and

the realization route of application-oriented talents cultivation in local colleges and Universities

[J].China Electric Power Education.2012,(12):11-12.

[11] CHEN Zhi-wu, ZHANG De-rong.Perfecting Practice-teaching System and cultivating creative

and application-oriented talents[J].Research and Exploration in Laboratory.2012,(8):167-170.

[12] PAN Mao-yuan.The Quality Concept of the Popular Higher Education[J].Jiangsu Higher 
Education.2000,(1):6-9.

[13] Chinese Education Science Research Institute International Comparative Education Research Centre.The Choice of the Time:Establishment of national standard of Education

Education.2012,(11):4-7. Quality[J].People's

[14] XIN Tao, JIANG Yu. The definition of educational quality standard under the international horizon. People's Education[J].2012,(12):5-9.

[15] YAO Lin-qun, GUO Yuan-xiang.Investigation of teaching quality standard based on the level

of school[J].Journal of The Chinese Society of Education.2012,(6):49-51. 